PROCEEDINGS OF THE

AMERICAN MATHEMATICAL SOCIETY

Volume 135, Number 5, May 2007, Pages 1329-1333

S 0002-9939(06)08777-6

Article electronically published on December 28, 2006

\title{
A GENERALIZATION OF A CURIOUS CONGRUENCE ON HARMONIC SUMS
}

\author{
XIA ZHOU AND TIANXIN CAI
}

(Communicated by Wen-Ching Winnie Li)

ABStRaCt. Zhao established a curious harmonic congruence for prime $p>3$ :

$$
\sum_{\substack{i+j+k=p \\ i, j, k>0}} \frac{1}{i j k} \equiv-2 B_{p-3}(\bmod p) .
$$

In this note the authors extend it to the following congruence for any prime $p>3$ and positive integer $n \leq p-2$ :

$$
\sum_{\substack{l_{1}+l_{2}+\cdots+l_{n}=p \\ l_{1}, \cdots, l_{n}>0}} \frac{1}{l_{1} l_{2} \cdots l_{n}} \equiv \begin{cases}-(n-1) ! B_{p-n}(\bmod p) & \text { if } 2 \nmid n, \\ -\frac{n}{2(n+1)} n ! B_{p-n-1} p\left(\bmod p^{2}\right) & \text { if } 2 \mid n .\end{cases}
$$

Other improvements on congruences of harmonic sums are also obtained.

\section{INTRODUCTION}

Bernoulli numbers $B_{k}(k=0,1,2, \ldots)$ is defined by the series $\frac{t}{e^{t}-1}=\sum_{k=0}^{\infty} B_{k} \frac{t^{k}}{k !}$. It is well known that $B_{0}=1, B_{1}=-\frac{1}{2}, B_{2}=\frac{1}{6}, B_{2 k+1}=0(k>0)$. The congruences concerning Bernoulli numbers have been studied by various authors. Using partial sum of multiple zeta value series, Jianqiang Zhao [7] first gave the congruence

$$
\sum_{\substack{i+j+k=p \\ i, j, k>0}} \frac{1}{i j k} \equiv-2 B_{p-3}(\bmod p)
$$

for any prime $p>3$.

Recently, Chungang Ji [1] gave a simple proof of the above congruence. Inspired by their work, we derive the following congruence.

Main Theorem. Let $p>3$ be a prime, $n \leq p-2$ a positive integer,

$$
\sum_{\substack{l_{1}+l_{2}+\cdots+l_{n}=p \\ l_{1}, \cdots, l_{n}>0}} \frac{1}{l_{1} l_{2} \cdots l_{n}} \equiv \begin{cases}-(n-1) ! B_{p-n}(\bmod p) & \text { if } 2 \nmid n, \\ -\frac{n}{2(n+1)} n ! B_{p-n-1} p\left(\bmod p^{2}\right) & \text { if } 2 \mid n .\end{cases}
$$

Received by the editors December 14, 2005 and, in revised form, February 6, 2006.

2000 Mathematics Subject Classification. Primary 11A07, 11A41.

Key words and phrases. Bernoulli numbers, congruences of harmonic sums, partitions.

This work was supported by the National Natural Science Foundation of China, Project 10371107 . 


\section{Several LEMmas}

Lemma 1 (Von Staudt-Clausen; cf. [6, p. 56]). Let $m$ be a positive integer. Then

$$
B_{2 m}+\sum_{p-1 \mid 2 m} \frac{1}{p} \in \mathbb{Z}
$$

where the sum is taken over those primes $p$ such that $p-1$ divides $2 m$ (in particular, 2 and 3 appear in the denominator of each Bernoulli number). Consequently, $p B_{2 m}$ is $p$-integral for all $m$ and all $p$.

Lemma 2 (cf. 2], 3], 4] or [5]). Let $p$ be an odd prime $>3$ and let $r$ be a positive integer, $r \leq p-3$. Then

$$
\sum_{i=1}^{p-1} \frac{1}{i^{r}} \equiv \begin{cases}-\frac{r(r+1)}{2(r+2)} B_{p-r-2} p^{2}\left(\bmod p^{3}\right) & \text { if } 2 \nmid r, \\ \frac{r}{r+1} B_{p-r-1} p\left(\bmod p^{2}\right) & \text { if } 2 \mid r .\end{cases}
$$

Lemma 3. Let $r, \alpha_{1}, \cdots, \alpha_{n}$ be positive integers, $r=\alpha_{1}+\alpha_{2}+\cdots+\alpha_{n} \leq p-3$. Then

$$
\sum_{\substack{1 \leq l_{1}, \cdots, l_{n} \leq p-1 \\ l_{i} \neq l_{j}}} \frac{1}{l_{1}^{\alpha_{1}} l_{2}^{\alpha_{2}} \cdots l_{n}^{\alpha_{n}}} \equiv \begin{cases}(-1)^{n}(n-1) ! \frac{r(r+1)}{2(r+2)} B_{p-r-2} p^{2}\left(\bmod p^{3}\right) & \text { if } 2 \nmid r, \\ (-1)^{n-1}(n-1) ! \frac{r}{r+1} B_{p-r-1} p\left(\bmod p^{2}\right) & \text { if } 2 \mid r .\end{cases}
$$

Proof. If $n=1$, the congruence is obvious by Lemma 2. We assume that the formula holds when the number of variables is less than $n$. Then by induction

$$
\begin{aligned}
& \sum_{\substack{1 \leq l_{1}, \cdots, l_{n} \leq p-1 \\
l_{i} \neq l_{j}}} \frac{1}{l_{1}^{\alpha_{1}} l_{2}^{\alpha_{2}} \cdots l_{n}^{\alpha_{n}}} \\
& =\sum_{\substack{1 \leq l_{1}, \cdots, l_{n-1} \leq p-1 \\
l_{i} \neq l_{j}}} \frac{1}{l_{1}^{\alpha_{1}} l_{2}^{\alpha_{2}} \cdots l_{n-1}^{\alpha_{n-1}}}\left(\sum_{l_{n}=1}^{p-1} \frac{1}{l_{n}^{\alpha_{n}}}-\frac{1}{l_{1}^{\alpha_{n}}}-\cdots-\frac{1}{l_{n-1}^{\alpha_{n}}}\right) \\
& =\sum_{\substack{1 \leq l_{1}, \cdots, l_{n-1} \leq p-1 \\
l_{i} \neq l_{j}}} \frac{1}{l_{1}^{\alpha_{1}} l_{2}^{\alpha_{2}} \cdots l_{n-1}^{\alpha_{n}-1}} \sum_{l_{n}=1}^{p-1} \frac{1}{l_{n}^{\alpha_{n}}} \\
& -\sum_{\substack{1 \leq l_{1}, \cdots, l_{n-1} \leq p-1 \\
l_{i} \neq l_{j}}} \frac{1}{l_{1}^{\alpha_{1}+\alpha_{n}} l_{2}^{\alpha_{2}} \cdots l_{n-1}^{\alpha_{n}-1}} \\
& -\cdots-\sum_{\substack{1 \leq l_{1}, \cdots, l_{n-1} \leq p-1 \\
l_{i} \neq l_{j}}} \frac{1}{l_{1}^{\alpha_{1}+\alpha_{n}} l_{2}^{\alpha_{2}} \cdots l_{n-1}^{\alpha_{n-1}+\alpha_{n}}} .
\end{aligned}
$$

From Lemma 1, Lemma 2 and the above assumption, we have

$$
\sum_{\substack{1 \leq l_{1}, \cdots, l_{n-1} \leq p-1 \\ l_{i} \neq l_{j}}} \frac{1}{l_{1}^{\alpha_{1}} l_{2}^{\alpha_{2}} \cdots l_{n-1}^{\alpha_{n-1}}} \sum_{l_{n}=1}^{p-1} \frac{1}{l_{n}^{\alpha_{n}}} \equiv \begin{cases}0\left(\bmod p^{3}\right) & \text { if } 2 \nmid r, \\ 0\left(\bmod p^{2}\right) & \text { if } 2 \mid r .\end{cases}
$$


If $r$ is odd, then,

$$
\begin{aligned}
\sum_{\substack{1 \leq l_{1}, \cdots, l_{n} \leq p-1 \\
l_{i} \neq l_{j}}} \frac{1}{l_{1}^{\alpha_{1}} l_{2}^{\alpha_{2}} \cdots l_{n}^{\alpha_{n}}} & \equiv-(n-1) \sum_{\substack{1 \leq l_{1}, \cdots, l_{n-1} \leq p-1 \\
l_{i} \neq l_{j}}} \frac{1}{l_{1}^{\alpha_{1}+\alpha_{n}} l_{2}^{\alpha_{2}} \cdots l_{n-1}^{\alpha_{n-1}}} \\
& \equiv-(n-1)(-1)^{n-1}(n-2) ! \frac{r(r+1)}{2(r+2)} B_{p-r-2} p^{2} \\
& \equiv(-1)^{n}(n-1) ! \frac{r(r+1)}{2(r+2)} B_{p-r-2} p^{2}\left(\bmod p^{3}\right),
\end{aligned}
$$

and if $r$ is even, we can similarly derive that

$$
\sum_{\substack{1 \leq l_{1}, \cdots, l_{n} \leq p-1 \\ l_{i} \neq l_{j}}} \frac{1}{l_{1}^{\alpha_{1}} l_{2}^{\alpha_{2}} \cdots l_{n}^{\alpha_{n}}} \equiv(-1)^{n-1}(n-1) ! \frac{r}{r+1} B_{p-r-1} p\left(\bmod p^{2}\right) .
$$

The proof of Lemma 3 is complete by induction.

\section{The proof of the Main Theorem}

Since $n \leq p-2$, it is easy to see that

$$
\begin{aligned}
& \sum_{\substack{l_{1}+l_{2}+\cdots+l_{n}=p \\
l_{1}, \cdots, l_{n}>0}} \frac{1}{l_{1} l_{2} \cdots l_{n}} \\
& \quad=\frac{1}{p} \sum_{\substack{l_{1}+l_{2}+\cdots+l_{n}=p \\
l_{1}, \cdots, l_{n}>0}} \frac{l_{1}+l_{2}+\cdots+l_{n}}{l_{1} l_{2} \cdots l_{n}}=\frac{n}{p} \sum_{\substack{l_{1}+l_{2}+\cdots+l_{n-1}<p \\
l_{1}, \cdots, l_{n-1}>0}} \frac{1}{l_{1} l_{2} \cdots l_{n-1}}
\end{aligned}
$$

Writing $u_{n-1}=l_{1}+l_{2}+\cdots+l_{n-1}$, so that $1 \leq l_{1}+l_{2}+\cdots+l_{n-2}<u_{n-1} \leq p-1$, we find that

$$
\frac{1}{l_{1} l_{2} \cdots l_{n-1}}=\frac{l_{1}+l_{2}+\cdots+l_{n-1}}{l_{1} l_{2} \cdots l_{n-1} u_{n-1}}=\frac{1}{u_{n-1}}\left(\frac{1}{l_{1} \cdots l_{n-2}}+\cdots+\frac{1}{l_{2} \cdots l_{n-1}}\right)
$$

and hence

$$
\sum_{\substack{l_{1}+l_{2}+\cdots+l_{n-1}<p \\ l_{1}, \cdots, l_{n-1}>0}} \frac{1}{l_{1} l_{2} \cdots l_{n-1}}=(n-1) \sum_{\substack{1 \leq l_{1}+l_{2}+\cdots+l_{n-2}<u_{n-1} \leq p-1 \\ l_{1}, \cdots, l_{n-2}>0}} \frac{1}{l_{1} l_{2} \cdots l_{n-2} u_{n-1}}
$$


Using this method step by step and denoting $l_{1}+\cdots+l_{k}=u_{k}$ we have

$$
\begin{aligned}
& \sum_{\substack{l_{1}+l_{2}+\cdots+l_{n}=p \\
l_{1}, \cdots, l_{n}>0}} \frac{1}{l_{1} l_{2} \cdots l_{n}} \frac{n}{p} \sum_{\substack{l_{1}+l_{2}+\cdots+l_{n-1}<p \\
l_{1}, \cdots, l_{n-1}>0}} \frac{1}{l_{1} l_{2} \cdots l_{n-1}} \\
& =\frac{n(n-1)}{p} \sum_{\substack{1 \leq l_{1}+l_{2}+\cdots+l_{n-2}<u_{n-1} \leq p-1 \\
l_{1}, \cdots, l_{n-2}>0}} \frac{1}{l_{1} l_{2} \cdots l_{n-2} u_{n-1}} \\
& =\cdots \\
& =\frac{n !}{p} \sum_{1 \leq u_{1}<u_{2}<\cdots<u_{n-1}<p} \frac{1}{u_{1} u_{2} \cdots u_{n-1}} \\
& =\frac{n !}{p} \frac{1}{(n-1) !} \sum_{\substack{1 \leq u_{1}, \cdots, u_{n-1} \leq p-1 \\
u_{i} \neq u_{j}}} \frac{1}{u_{1} u_{2} \cdots u_{n-1}} \\
& =\frac{n}{p} \sum_{\substack{1 \leq u_{1}, \cdots, u_{n-1} \leq p-1 \\
u_{i} \neq u_{j}}} \frac{1}{u_{1} u_{2} \cdots u_{n-1}} .
\end{aligned}
$$

From Lemma 3,

$$
\begin{aligned}
& \sum_{\substack{1 \leq u_{1}, \cdots, u_{n-1} \leq p-1 \\
u_{i} \neq u_{j}}} \frac{1}{u_{1} u_{2} \cdots u_{n-1}} \\
& \equiv \begin{cases}(-1)^{n-1}(n-1) ! \frac{n}{2(n+1)} B_{p-n-1} p^{2}\left(\bmod p^{3}\right) & \text { if } 2 \nmid(n-1), \\
(-1)^{n-2}(n-1) ! \frac{1}{n} B_{p-n} p\left(\bmod p^{2}\right) & \text { if } 2 \mid(n-1),\end{cases}
\end{aligned}
$$

we have

$$
\sum_{\substack{l_{1}+l_{2}+\cdots+l_{n}=p \\ l_{1}, \cdots, l_{n}>0}} \frac{1}{l_{1} l_{2} \cdots l_{n}} \equiv \begin{cases}-n ! \frac{n}{2(n+1)} B_{p-n-1} p\left(\bmod p^{2}\right) & \text { if } 2 \mid n, \\ -(n-1) ! B_{p-n}(\bmod p) & \text { if } 2 \nmid n .\end{cases}
$$

We have completed the proof of the Main Theorem.

Corollary (Zhao). For any prime $p>3$,

$$
\sum_{\substack{i+j+k=p \\ i, j, k>0}} \frac{1}{i j k} \equiv-2 B_{p-3}(\bmod p) .
$$

Remark. In [8] Zhao also considered another congruence on harmonic sum. For any positive integer $n$ and $\alpha, n \alpha \leq p-3$, he obtains

$$
\sum_{1 \leq l_{1}<l_{2}<\cdots<l_{n} \leq p-1} \frac{1}{l_{1}^{\alpha} l_{2}^{\alpha} \cdots l_{n}^{\alpha}} \equiv 0 \begin{cases}\left(\bmod p^{2}\right) & \text { if } 2 \nmid n \alpha, \\ (\bmod p) & \text { if } 2 \mid n \alpha .\end{cases}
$$

By using Lemma 3, we improve the above congruence to

$$
\sum_{1 \leq l_{1}<l_{2}<\cdots<l_{n} \leq p-1} \frac{1}{l_{1}^{\alpha} l_{2}^{\alpha} \cdots l_{n}^{\alpha}} \equiv \begin{cases}(-1)^{n} \frac{\alpha(n \alpha+1)}{2(n \alpha+2)} B_{p-n \alpha-2} p^{2}\left(\bmod p^{3}\right) & \text { if } 2 \nmid n \alpha, \\ (-1)^{n-1} \frac{\alpha}{n \alpha+1} B_{p-n \alpha-1} p\left(\bmod p^{2}\right) & \text { if } 2 \mid n \alpha .\end{cases}
$$

for any positive integers $n$ and $\alpha$ with $n \alpha \leq p-3$, since the sum is over ordered $l_{i}^{\prime}$ 's. 


\section{ACKNOWLEDGMENTS}

The authors thank the referee for his constructive suggestion and careful reading of the manuscript, and for bringing the paper [8] to our attention.

\section{REFERENCES}

[1] Chun-Gang Ji, A Simple Proof of A Curious Congruence By Zhao. Proceedings of The American Mathematical Society, 133 (2005):3469-3472. MR 2163581 (2006d:11005)

[2] J.W.L. Glaisher, On the residues of the sums of products of the first p-1 numbers, and their powers, to modulus $p^{2}$ or $p^{3}$. Quart. J. Pure Appl. Math., 31 (1900): 321-353.

[3] J.W.L. Glaisher, On the residues of the inverse powers of numbers in arithmetic progression. Quart. J. Pure Appl. Math., 32 (1901):271-305.

[4] E. Lehmer, On congruences involving Bernoulli numbers and the quotients of Fermat and Wilson. Ann. Math., 39 (1938):350-360. MR 1503412

[5] Zhihong Sun, Congruence concerning Bernoulli numbers and Bernoulli polynomials, Discrete Applied Mathematics, 105 (2000):193-223. MR1780472 (2001m:11022)

[6] L.C. Washington, Introduction to Cyclotomic Fields, 2nd ed., Springer-Verlag, New York, 1997. MR1421575 (97h:11130)

[7] Jiangqiang Zhao, Bernoulli numbers, Wolstenholme's Theorem, and $p^{5}$ variations of Lucas' Theorem, arxiv.org/abs/math.NT/0303332

[8] Jiangqiang Zhao, Multiple Harmonic Sums I: Generalizations of Wolstenholme's Theorem, xxx.lanl.gcv/abs/math.NT/0301252

Department of Mathematics, Zhejiang University, Hangzhou 310027, People's RepubLIC OF CHINA

E-mail address: unitqq@zju.edu.cn

Department of Mathematics, Zhejiang University, Hangzhou 310027, People's RepubLIC OF CHINA

E-mail address: txcai@mail.hz.zj.cn 